\title{
Classifying kinase conformations using a machine learning approach
}

\author{
Daniel lan McSkimming ${ }^{1}$, Khaled Rasheed ${ }^{2}$ and Natarajan Kannan ${ }^{1,3^{*}}$
}

\begin{abstract}
Background: Signaling proteins such as protein kinases adopt a diverse array of conformations to respond to regulatory signals in signaling pathways. Perhaps the most fundamental conformational change of a kinase is the transition between active and inactive states, and defining the conformational features associated with kinase activation is critical for selectively targeting abnormally regulated kinases in diseases. While manual examination of crystal structures have led to the identification of key structural features associated with kinase activation, the large number of kinase crystal structures $(\sim 3,500)$ and extensive conformational diversity displayed by the protein kinase superfamily poses unique challenges in fully defining the conformational features associated with kinase activation. Although some computational approaches have been proposed, they are typically based on a small subset of crystal structures using measurements biased towards the active site geometry.

Results: We utilize an unbiased informatics based machine learning approach to classify all eukaryotic protein kinase conformations deposited in the PDB. We show that the orientation of the activation segment, measured by $\varphi, \psi, \times 1$, and pseudo-dihedral angles more accurately classify kinase crystal conformations than existing methods. We show that the formation of the K-E salt bridge is statistically dependent upon the activation segment orientation and identify evolutionary differences between the activation segment conformation of tyrosine and serine/threonine kinases. We provide evidence that our method can identify conformational changes associated with the binding of allosteric regulatory proteins, and show that the greatest variation in inactive structures comes from kinase group and family specific side chain orientations.

Conclusion: We have provided the first comprehensive machine learning based classification of protein kinase active/ inactive conformations, taking into account more structures and measurements than any previous classification effort. Further, our unbiased classification of inactive structures reveals residues associated with kinase functional specificity. To enable classification of new crystal structures, we have made our classifier publicly accessible through a stand-alone program housed at https://github.com/esbg/kinconform [DOl:10.5281/zenodo.249090].
\end{abstract}

Keywords: Kinase conformation, Machine learning, Classifier, Activation segment

\section{Background}

Protein kinases are a diverse family of signaling proteins whose catalytic activity is involved in nearly all cellular processes. The enzymatic activity of kinases is regulated through conformational changes in the protein kinase domain, which is shared by diverse members of the protein kinase super-family [1-3]. Structural studies on members of the protein kinase superfamily have shown

\footnotetext{
* Correspondence: nkannan@uga.edu

${ }^{1}$ Institute of Bioinformatics, University of Georgia, Athens, GA 30602, USA ${ }^{3}$ Department of Biochemistry \& Molecular Biology, University of Georgia, Athens, GA 30602, USA

Full list of author information is available at the end of the article
}

that the protein kinase domain is malleable and can undergo dramatic conformational changes in response to activation and regulatory signals in signaling pathways. The conformation of protein kinases is controlled by factors including protein-protein interactions [4], phosphorylation and ligand binding $[5,6]$, and numerous drug discovery efforts on protein kinases are focused on targeting specific kinase conformations [7-11]. However, an incomplete understanding of the defining conformational features of protein kinases and how these differ between kinase groups and families has hindered ongoing drug discovery efforts to improve inhibitor specificity. 
The manual inspection of kinase crystal structures has led to an understanding of the roles key residues play in stabilizing and orienting adenosine tri-phosphate (ATP) for phosphoryl transfer $[12,13]$, as well as qualitative descriptions of the active site geometry and conformational states. A variety of structural measures have been developed to determine the activation state of a kinase, most of which center on the orientation of two regulatory components: the $\alpha \mathrm{C}$-helix and the activation segment. The $\alpha \mathrm{C}$-helix serves as a proxy for the formation of a catalytically crucial salt bridge between a lysine in the $\beta 3$ strand and the $\alpha C$ helix glutamate (K-E salt bridge), which positions the lysine to anchor the $\alpha$-phosphate of ATP (Fig. 1a). The activation segment provides two pieces of information: the orientation of the DFG aspartate, which chelates a magnesium ion that coordinates with the $\beta$ - and $\gamma$-phosphates of ATP (Fig. 1b), and whether the $\mathrm{C}$-terminal activation segment is blocking the substrate binding site (Fig. 1c).

The orientation of the DFG phenylalanine, a proxy for the DFG aspartate located in the $\mathrm{N}$-terminal activation segment, is of particular importance for inhibitor design, as the DFG-out conformation creates a hydrophobic pocket that can be targeted by Type II ATP competitive inhibitors $[7,8,14-16]$. Studies focused on the classification of active site conformation for drug discovery purposes have generally used manual curation [17] or constructed complex template based metrics specific for that purpose [18]. For example, in Kufareva and
Abagyan, the authors chose a template DFG-in conformation in the Abelson tyrosine kinase (Abl1) structure [PDB: 2GQG] to generate a DFG-Phe orientation index, $\mathrm{O}_{\text {phe, }}$, by summing the cosines of angles between covalent bonds $\left(C \alpha-C \beta, C \beta-C \gamma, C \gamma-C \delta^{1}, C \gamma-C \delta^{2}\right)$ in the template and aligned structures. The position, $\mathrm{P}_{\text {phe, was }}$ calculated as the distance between the $\mathrm{C} \alpha$ atoms in the template and aligned DFG-Phe residue, with the final DFG-in score $\left(\mathrm{S}_{\mathrm{DFG}-\mathrm{in}}\right)$ defined as a function of $\mathrm{O}_{\text {phe }}$ and $\mathrm{P}_{\text {phe. }}$ A hard cutoff of $\mathrm{S}_{\mathrm{DFG} \text {-in }}$ was used to classify DFGin versus DFG-out conformations. Methods that consider activation segment conformation to classify kinase structures have been reported as well. One such method used a comparison to the cAMP-dependent protein kinase catalytic subunit $\alpha(\mathrm{PKA} \alpha)$ structure [PDB:1BKX], where root mean squared deviation (RMSD) calculations and counting atoms separated by predefined planes determined the conformational state of the $\alpha \mathrm{C}$-helix and activation segments, respectively, with hard cutoffs distinguishing active from inactive kinases [19]. Other methods have benefitted from exponential increases in published kinase structures, though limited themselves to measuring the $\alpha \mathrm{C}$-helix and DFG motif conformations. In one (Brooijmans'), the distance between the side chain nitrogen of the $\beta 3$ lysine and the $\alpha \mathrm{C}$-helix glutamate's terminal oxygen's were used to measure the orientation of the $\alpha \mathrm{C}$-helix and the K-E salt bridge distance, using a hard cutoff of $4 \AA$. The activation segment

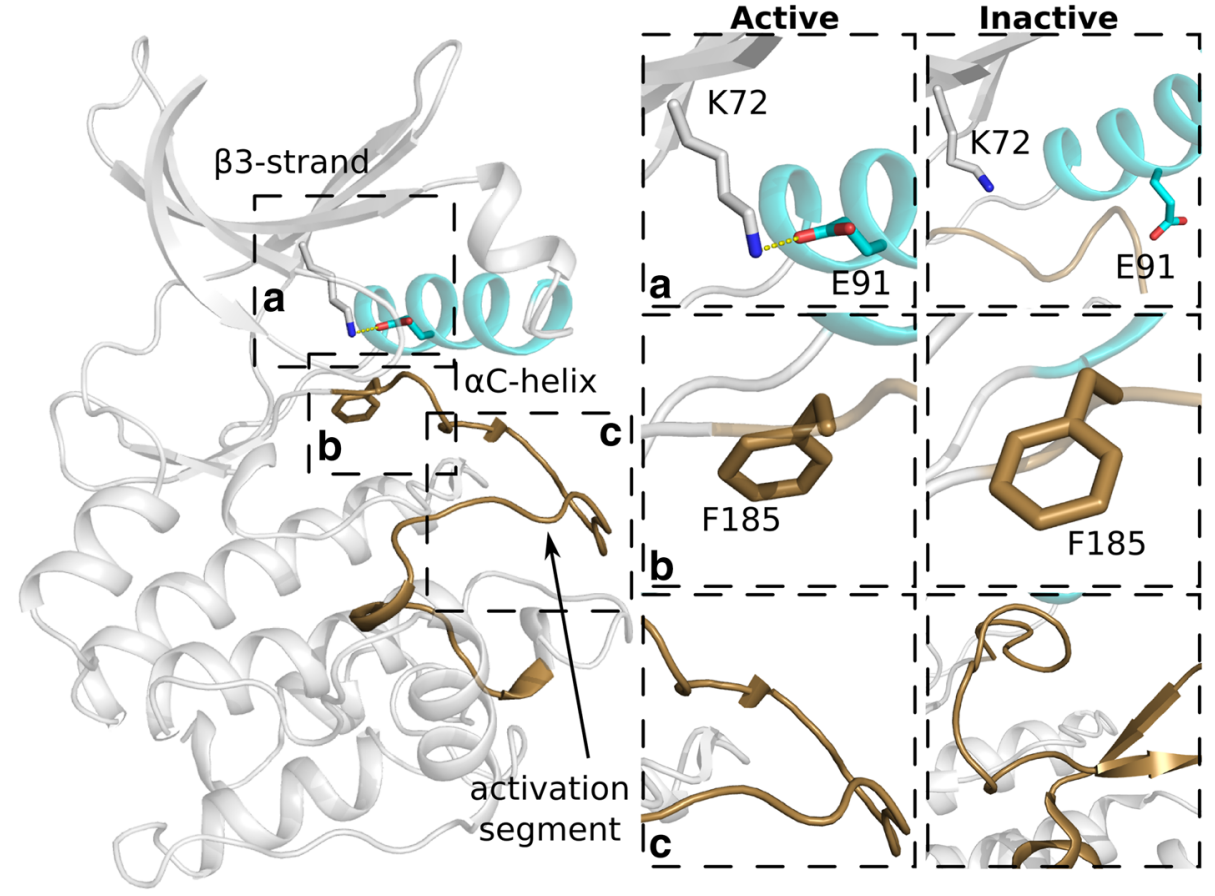

Fig. 1 Kinase activation. a Formation of the K-E salt bridge (left). The salt bridge is not formed in the inactive conformation (right). b DFG-in (left) and DFG-out (right) conformations. c The inactive activation segment (right) can occlude the substrate binding region. The aC-helix is colored teal and the activation segment is colored brown 
orientation was measured using the distance between the main chain nitrogen in the hinge donor residue and the C $\alpha$ atom in the DFG-Phe, with a soft cutoff of $15 \AA$ [20]. In another, the $\alpha C$-helix orientation (in/out/outlike) was determined using the distance between the $\mathrm{C} \alpha$ atoms of the DFG aspartate and the $\alpha \mathrm{C}$-helix glutamate, while the DFG motif orientation was manually curated [21]. The most recent approach (ABC method) notes that the DFG orientation is captured more accurately with pseudo-dihedral angles, or dihedrals through adjacent quads of $\mathrm{C} \alpha$ atoms [22]. Multiple conformational states for both the DFG motif and the $\alpha$ C-helix are defined, but the active conformation is only attained when both the DFG-in and $\alpha \mathrm{C}$-helix-in orientations are present. The $\alpha \mathrm{C}$-helix orientation is defined using the distance between the $\mathrm{C} \alpha$ atom of the $\alpha \mathrm{C}$-helix glutamate and the $\mathrm{C} \alpha$ atom of the DFG-Phe, with the $\alpha C$-helix-in conformation occurring when this distance is less than $10.5 \AA$. The DFG orientation is measured using three pseudo-dihedral angles, with the DFG-in state defined when all three pseuo-dihedrals are within specified ranges.

Perhaps the most interesting classification method, as it was the first method published and does not explicitly measure any angles or distances, is the formation of the hydrophobic Regulatory spine (R-spine) [23, 24], discovered through the surface comparison of 23 kinase structures. The formation of hydrophobic interactions between the $\mathrm{N}$ - and $\mathrm{C}$-lobes of the kinase domain are described as characteristic of active structures, with inactive structures breaking the spine in four ways [24]. While this method is highly qualitative and difficult to measure, the spine formation is mechanistically explained, with the assembly of the spine coordinated with other conformational changes associated with kinase activation such as the formation of the K-E salt bridge and the relative orientation of the catalytically important DFG and HRD motifs [23]. Though the precise order in which these interactions develop is unknown, the assembly of the R-spine is highly dependent on the orientation of the activation segment [25].
While the above methods have provided insights into kinase conformational states, they are limited in several ways. Many only consider a small subset of the 5,131 kinase chains deposited in the PDB, thereby leaving out valuable information. Some studies have used a large subset of structures/conformations, but only a limited number of features, with emphasis placed on the active site orientation [19-23]. These limitations lead to conflicting assessments and, in some cases, misannotation of kinase conformations. For example, while a broken Rspine is characteristic of an inactive kinase, an assembled $\mathrm{R}$-spine does not necessarily reflect an active conformation. In the epidermal growth factor receptor (EGFR) structure [PDB:2GS7] for example, the R-spine is assembled, but the activation segment is in an inactive conformation with a disrupted K-E salt bridge (Fig. 2a). Distance measures are problematic when the endpoint atoms are in dynamic loop regions. The active checkpoint kinase 1 (Chk1) structure [PDB:2AYP], which has an unusual linker conformation, is incorrectly annotated as inactive using Brooijmans' method (Fig. 2b). The most recently published $A B C$ method, which uses hard cutoffs on angle measurements, also leads to improper annotations. For example, the RAF proto-oncogene serine/ threonine protein kinase (RAF1) structure [PDB:3OMV] is clearly active, with a well-established K-E salt bridge and DFG-in conformation, yet is annotated by $\mathrm{ABC}$ as inactive (Fig. 2c). In contrast, the inactive serine/threonine protein kinase B-raf (BRAF) structure [PDB:3SKC] is annotated by $A B C$ as active (Fig. 2d). Further, complex correlations between measurements can be difficult to identify and interpret without an appropriate statistical framework. Here, we take a systematic statistically based approach, using the automated pattern recognition algorithms in machine learning to identify the conformational changes between active and inactive protein kinases. We find that the orientation of the activation segment alone is sufficient to accurately classify kinase conformations as active or inactive, and identify the relative importance of different regions of the activation segment in classifying protein tyrosine kinase (PTK) and serine/threonine kinase

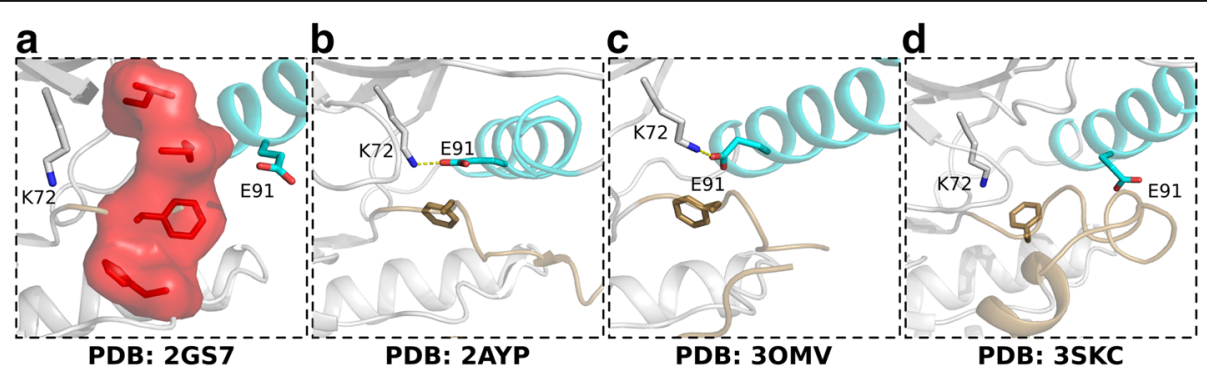

Fig. 2 Incorrectly classified structures. a Assembled R-spine without K-E salt bridge formed. b Structure improperly annotated using Brooijmans' method. c-d Structures improperly annotated using ABC method. The aC-helix is colored teal and the activation segment is colored brown 
(STK) conformations. We show the greatest variation between inactive structures results from evolutionary relationships between kinases, identifying a variety of residues that can be used to increase drug specificity. Finally, by applying our methods to the cyclin-dependent kinase family (CDKs), we identify interface residues associated with cyclin binding and recognition. While machine learning methods have been widely used in secondary structure and backbone torsion angle prediction from primary sequence [26-29], their use in structure based classification tasks has been limited to the identification of Structural Classification of Proteins (SCOP) domains and ligand prediction $[30,31]$. Only recently have machine learning techniques been applied to identify quantitative structure-activity relationships (QSARs) [32] or improve protein homology detection [33], though even these techniques are limited to using primary sequence input. When structures are used in machine learning algorithms, they are typically subjected to molecular dynamics simulations which are used to calculate a parameter of interest, such as energy landscapes [34] or thermostability [35]. We also provide access to the established classifier through a stand-alone program, allowing any user to classify PDB structures or models, and have deposited our training annotations and predictions in ProKinO, the Protein Kinase Ontology [36, 37].

\section{Results \& discussion}

Kinase conformation is determined by activation segment orientation

Previous methods have focused on the active site geometry for conformation classification, using the DFG motif and $\alpha \mathrm{C}$-helix orientations as proxies for the catalytically necessary placement of key residues. This active site focus naturally biases the proposed measurements by ignoring distal regions of the kinase domain. Instead, we developed features that encompass the entire domain and uniquely define each structure. Namely, for each residue in our profile based alignments, we incorporate the corresponding $\phi$, $\psi$ and $\chi^{1}$ angles. In addition, for each set of four consecutive residues, we calculate the pseudo-dihedral between their $\mathrm{C} \alpha$ atoms (Fig. 3a). Using our feature selection process on the described training set (see Methods), we rank ordered the 961 features, the top 15 of which are identified in Table 1. The regions identified are highly consistent between selection algorithms and identify measures describing the orientation of the activation segment. We divided the activation segment into three sections, based on their function and interacting regions. The most $\mathrm{N}$ terminal portion describes the orientation of the DFG motif, followed by a region which forms electrostatic and hydrophobic interactions with the $\alpha \mathrm{C}$-helix, and finally the most C-terminal portion of the activation segment, which can sterically block the binding of substrate (Fig. 3b).

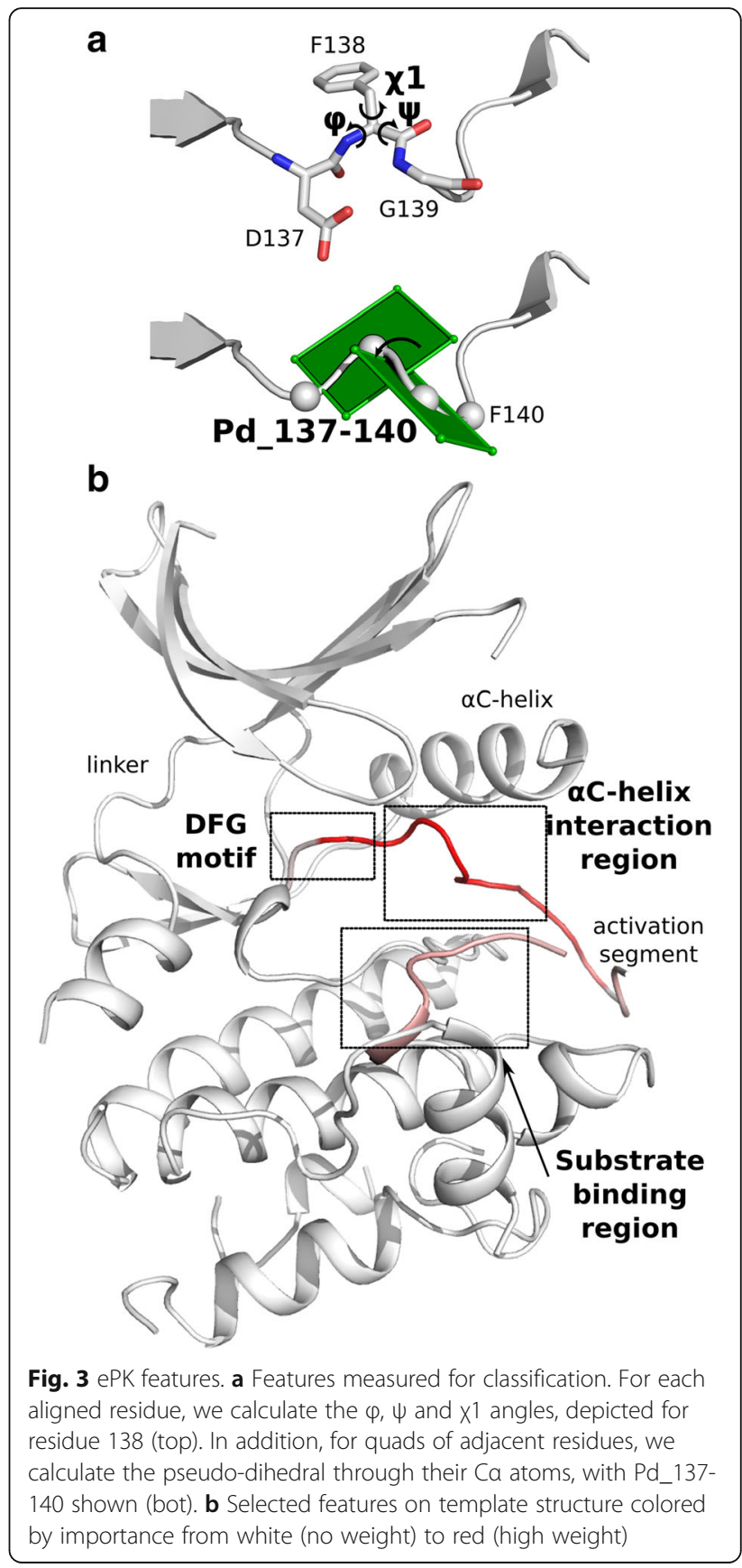

As coordinates of residues in the activation segment are commonly missing from crystal structures, regardless of conformation, we identified the frequency of activation segment residues without missing coordinates across the full dataset (Fig. 4a). To ensure we were not simply capturing the presence/absence of coordinates as a feature for classification, we only considered residues (and associated features) if they were present in more than $75 \%$ of the dataset. To assess the relative importance of the selected features, we initially trained a random forest classifier based on the most frequently chosen 
Table 1 Top 15 ePK selected features

\begin{tabular}{|c|c|c|c|}
\hline Feature & Activation segment location & PKA positions & Average rank \\
\hline Pd_137-140 & N-terminal & 184-187 & 2.88 \\
\hline Ф_141 & $\mathrm{N}$-terminal & 188 & 4.20 \\
\hline Pd_140-143 & N-terminal & 187-190 & 7.95 \\
\hline Pd_141-144 & N-terminal & 188-191 & 8.95 \\
\hline Ф_142 & N-terminal & 189 & 9.57 \\
\hline Ф_143 & $\mathrm{N}$-terminal & 190 & 10.15 \\
\hline U_139 & $\mathrm{N}$-terminal & 186 & 10.65 \\
\hline Ф_138 & N-terminal & 185 & 14.15 \\
\hline Pd_139-142 & $\mathrm{N}$-terminal & $186-189$ & 20.75 \\
\hline Pd_155-158 & C-terminal & $200-203$ & 22.15 \\
\hline Pd_138-141 & $\mathrm{N}$-terminal & $185-188$ & 27.00 \\
\hline Pd_135-138 & N-terminal & $182-185$ & 27.25 \\
\hline Pd_136-139 & N-terminal & $183-186$ & 30.02 \\
\hline Pd_154-157 & C-terminal & 199-202 & 31.02 \\
\hline Ф_142 & $\mathrm{N}$-terminal & 189 & 32.20 \\
\hline
\end{tabular}

feature, which captures the orientation of the DFG motif. Using this feature alone, we achieved over $90 \%$ classification accuracy, with precision and recall slightly higher and lower, respectively (Fig. 4b-c). The addition of the second feature, which describes the orientation of the activation segment following the DFG motif, increased our classification accuracy to $\sim 97 \%$. Subsequently, we trained additional classifiers with an increasing number of features, evaluating the accuracy increase associated with the incorporation of each new feature on classifying our training (Fig. 4b) and validation (Fig. 4c) sets.

We compared the performance of a variety of machine learning classifiers, the Brooijmans', $\mathrm{ABC}$, and R-spine methods using our validation set. As the criteria for determining the assembly of the R-spine is not well defined, we calculated the van der Waals (vdW) interactions between pairs of residues which are adjacent in the spine. If the $\mathrm{vdW}$ energy is less than $-1.5 \mathrm{kcal} / \mathrm{mol}$, we consider the spine to be assembled. This approach accurately identifies previously noted R-spine assembled structures [23]. Of the previously defined methods, the $\mathrm{R}$-spine assembly has the highest area under the curve (AUC) (0.736) and is able to classify the largest proportion of structures, missing only $6.7 \%$ (158 of 2,365 chains) of the validation set. The $\mathrm{ABC}$ method performs well when the necessary residues are ordered, but is unable to classify $18.8 \%$ (445 of 2,365 chains) of the validation set. The Brooijmans' method has lower performance when it is able to make an assessment, but only misses $8.2 \%$ (193 of 2,365 chains) of the validation set. Our machine learning classifiers, however, can assess $100 \%$ of the validation set, with all algorithms achieving classification accuracies greater than 97\% (Fig. 5a). Furthermore, we correctly

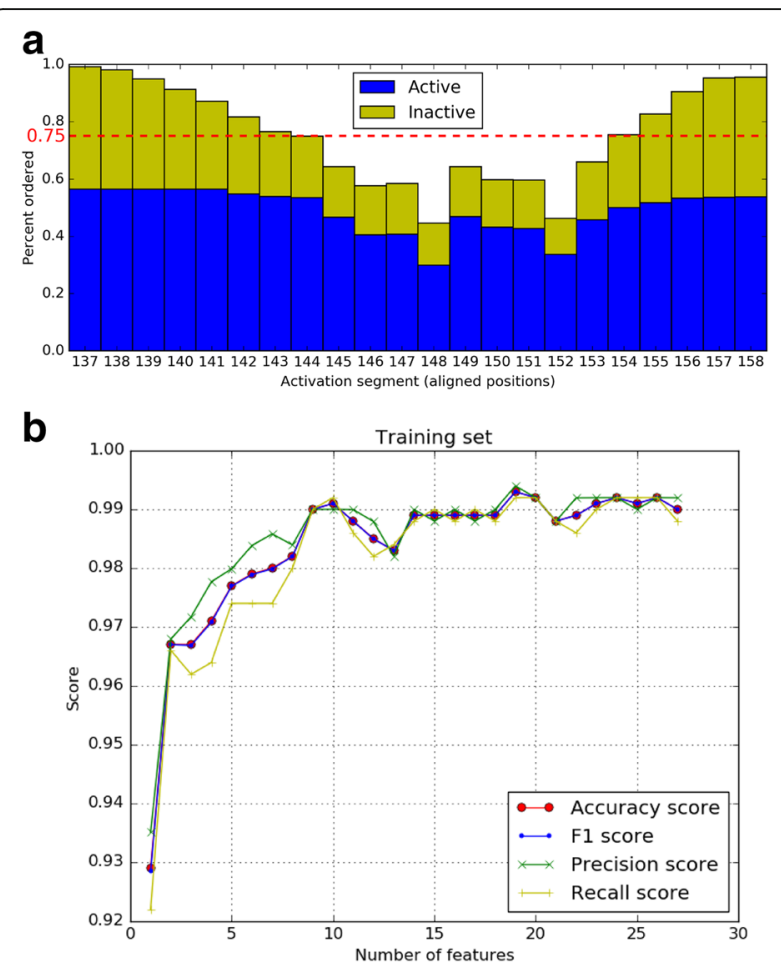

C

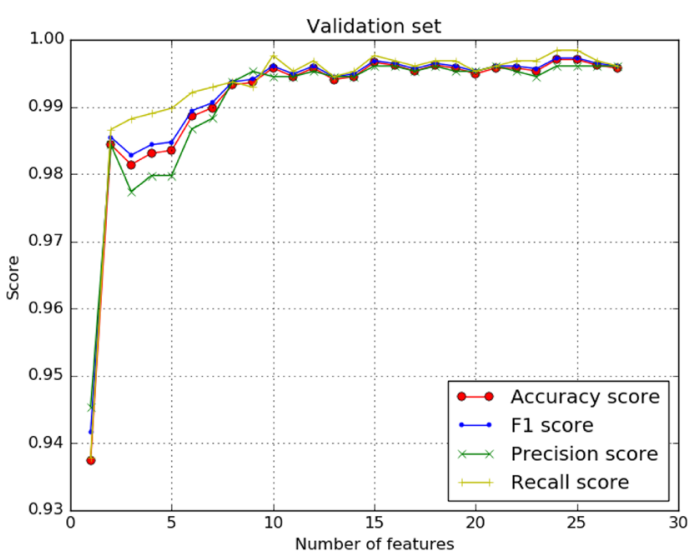

Fig. 4 Disorder and accuracy. a Chart showing the percentage of structures with activation segment disorder. b Accuracy with increasing number of features on training set. $\mathbf{c}$ Accuracy with increasing number of features on validation set

classify the EGFR [PDB:2GS7], Chk1 [PDB:2AYP], RAF1 [PDB:3OMV], and BRAF [PDB:3SKC] structures which were among those incorrectly annotated using the previous methods. While the generated classifiers can accurately predict the conformation of all the structures in our validation set, previous methods cannot make a prediction with missing atoms or residues (Fig. 5b). We chose the ensemble random forest classifier for the remainder of analyses, as it out performed all other algorithms.

Modification of the activation segment by phosphorylation is an important step in kinase activation and is required for most kinases to become fully active [6]. 

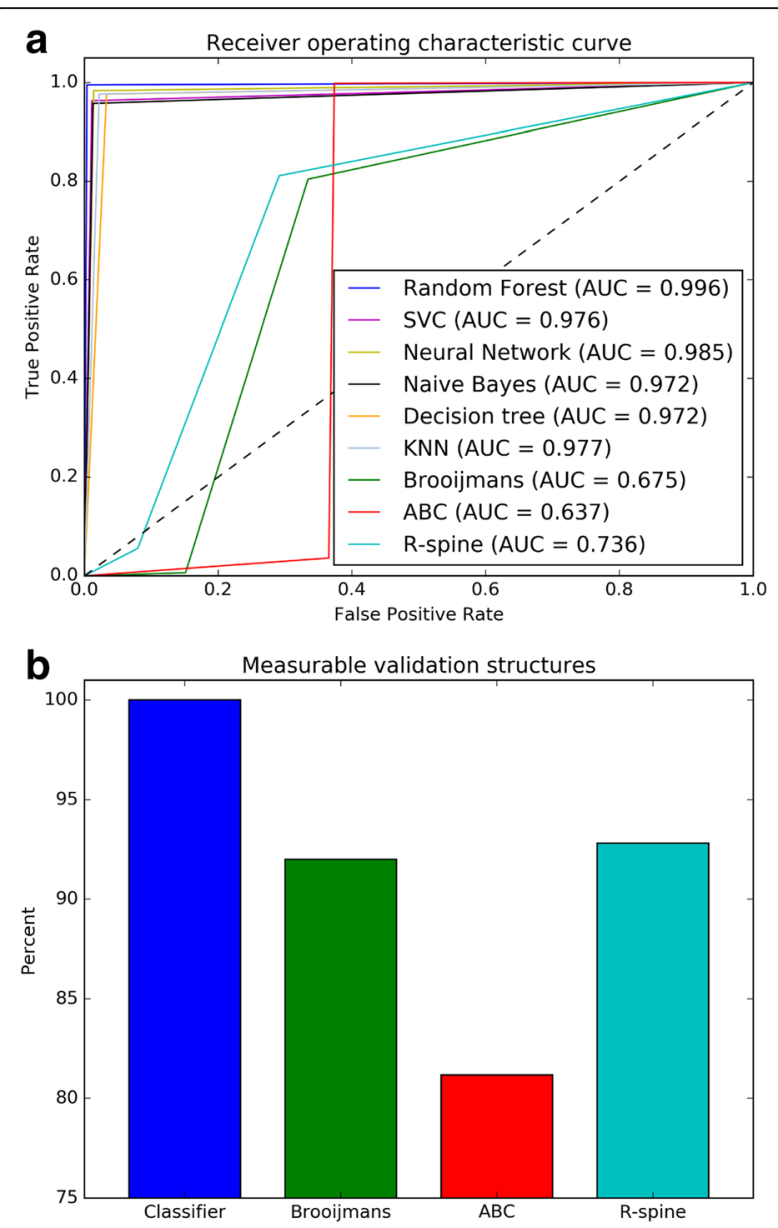

Fig. 5 a Receiver operating characteristic curve comparing classifiers and previous methods. $\mathbf{b}$ Percent of structures which can be measured and classified with various approaches

The conformational changes brought about by the phosphorylation event, which include ordering of the C-terminal activation segment for substrate binding, have been previously described [38-40]. The C-terminal activation segment, however, has not been used to assess kinase conformational state. Our identification of the Nterminal activation segment is consistent with previous classification models, particularly with our top selected feature which relates the orientation of the DFG-Phe to the conformational state, but also incorporates previously unidentified information concerning the orientation of the C-terminal activation segment. Nearly all validation structures were properly classified, leaving only 12 PDB chains whose curated annotation differed from the predicted conformational state. Manual examination of the 12 chains indicated that the difference is not due to machine learning classifier, but rather due to the misannotation of chains in the training set (Fig. 6). The chains are part of homo-dimeric complexes where one chain adopts an active conformation, and the other an inactive conformation, however, both chains were annotated as active or inactive based on manual evaluation of a single chain. For example, in the Mitogenactivated protein kinase 8 (JNK1) [PDB: 2GMX] in chain A, the K-E salt bridge is assembled (active), but not in chain B (inactive). However, both chains were annotated as active based on examination of chain A alone. Thus, the 12 chains were not actually misclassified, but rather the classifier had identified errors in our curated annotations.

\section{$\mathrm{K}-\mathrm{E}$ salt bridge formation is dependent on activation segment orientation}

Our classifier was trained using features specific to the orientation of the activation segment, a deviation from previous methods which also incorporated $\alpha \mathrm{C}$-helix orientation and the formation of the K-E salt bridge. The orientation of the loops preceding and following the $\alpha \mathrm{C}$ helix were captured in our initial feature set, providing information about the $\alpha \mathrm{C}$-helix orientation, but weren't identified by feature selection as important for classification. This suggests the $\alpha \mathrm{C}$-helix orientation, and hence the salt bridge formation, is dependent on the orientation of activation segment, which is captured in our selected features. To test this hypothesis, we used our trained ePK classifier to predict the conformational state of the remaining 1,766 unlabelled structures. As the features used correspond to the activation segment, we are essentially using only that portion of the kinase domain to distinguish active from inactive kinases. We also measured the distance between the side chain nitrogen atom in the beta- 3 lysine and oxygen atoms in the $\alpha C$-helix glutamate of the same structures. If the distance was less than $4 \AA$, we classified the structure as having an intact salt bridge (Table 2). We identified statistical correlations between the active-inactive classification and salt bridge formation, using both Fisher's exact and chi-squared tests. We find a strong dependence relationship between activation segment orientation and formation of the K-E salt bridge, with a $p$-value on the order of $10^{-49}$, explaining the lack of identified features measuring $\alpha \mathrm{C}$-helix orientation. We do not need to measure the $\alpha \mathrm{C}$-helix separately, as we gain the information by considering only the activation segment orientation.

We can also identify the $\alpha C$-helix/activation segment dependence through the visual inspection of crystal structures. When the kinase is in an active conformation, the DFG-phenylalanine adopts the in-conformation and the activation segment is pulled back, allowing the $\alpha$ C-helix to move in towards the active site and form the K-E salt bridge with the $\beta 3$ lysine (Fig. 7a). However, several inactive conformations of the activation segment can hinder $\alpha C$-helix movement. For example, the activation segment of inactive structures often forms a $1 \frac{1}{2}$ turn helix C- 


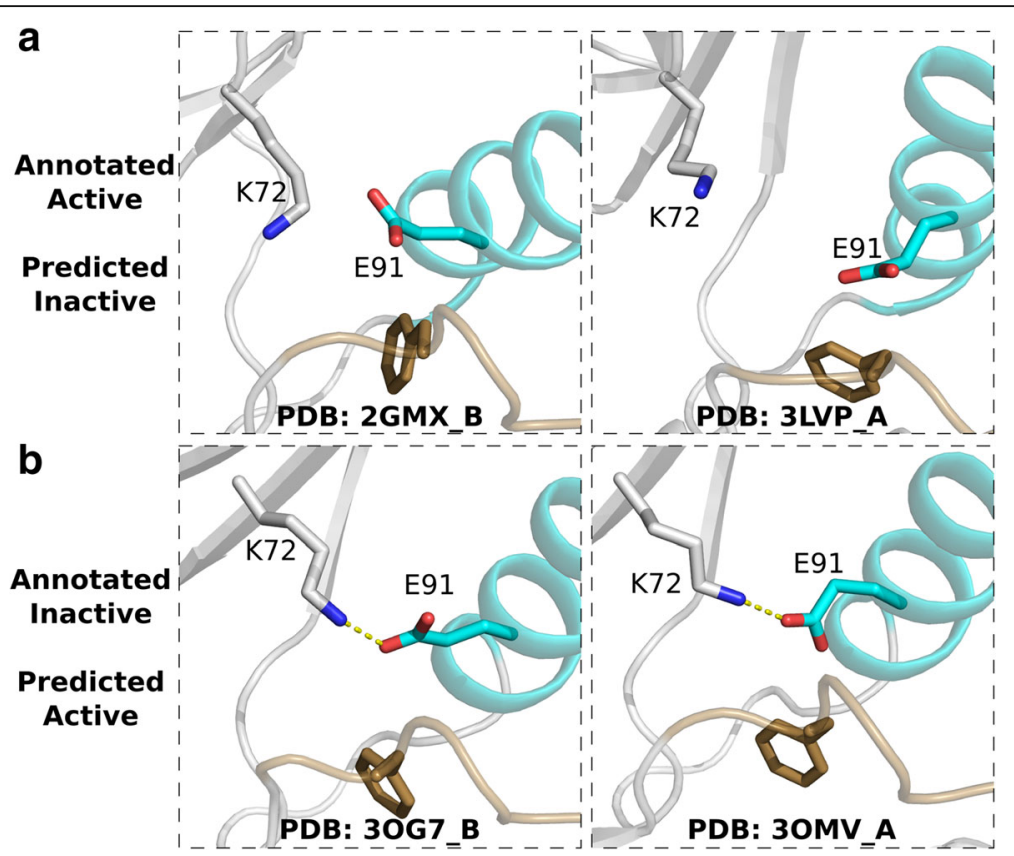

Fig. 6 Misannotated structures. a Structures misannotated as active which were properly identified as inactive by the classifier. $\mathbf{b}$ Structures misannotated as inactive were properly classified as active by the classifier. The aC-helix is colored teal and the activation segment is colored brown

terminal to the DFG motif, while the $\alpha \mathrm{C}$-helix is moved away from the active site, rotating the conserved glutamate towards the solvent (Fig. 7b). Here, the $1 \frac{1 / 2}{2}$ turn helix makes electrostatic and hydrophobic interactions with the displaced $\alpha C$-helix, stabilizing the inactive conformation. Alternatively, the DFG-phenylalanine can adopt an upconformation, which only slightly hinders $\alpha C$-helix movement yet sterically blocks the formation of the K-E salt bridge. In this situation, the $\alpha \mathrm{C}$-helix visually appears to be in the in-conformation and aligning to active structures shows little difference (Fig. 7c). It should be noted that KE salt bridge formation does not guarantee an active kinase, as the salt bridge can be formed in the DFG-out inactive conformation.

\section{PTK and STK conformations are distinguished by different} regions of the activation segment

Next, we wanted to apply our approach to evolutionarily related subsets of kinases. However, the number of solved structures is not uniformly distributed across the kinome, and some groups, families and subfamilies are not well represented. To ensure meaningful results, we first identified

Table 2 Testing dependence of activation segment orientation and formation of the K-E salt bridge

\begin{tabular}{lll}
\hline & K-E bridge & No K-E bridge \\
\hline Predicted active & 846 & 205 \\
Predicted inactive & 200 & 278 \\
\hline
\end{tabular}

$P$-value $<10^{-49}$ with Fisher's exact and chi-squared tests the number of training samples needed to construct an accurate classifier through the generation of learning curves, which train classifiers using an increasing number of data points and assesses the accuracy of each classifier. Fewer than 200 structures were needed as training data to classify the remainder with greater than $99 \%$ accuracy (Fig. 8). As we have 1,008 labeled protein tyrosine kinase (PTK) and 2,357 serine-threonine kinase (STK) chains, we applied our feature selection process to these sets.

In PTKs, we achieve a stable $>99 \%$ classification accuracy with the top 10 selected features (Table 3), all of which occur in the $\mathrm{N}$-terminal activation segment (Fig. 9a). STK classifiers, on the other hand, do not achieve stability in classification accuracy until $\sim 20$ features are incorporated (Table 4), with important C-terminal activation segment features identified (Fig. 9b). To ensure our STK measures are not dominated by a single group, since 46\% (1,090 of 2,357 ) of our STK chains belong to the CMGC group, a collection of kinases named after its four major members the CDK, MAPK, GSK3, and CLK families, we also performed feature selection on the non-CMGC STKs. Again, the top selected features corresponded to both the $\mathrm{N}$ - and C-terminal activation segments (Table 5). This is consistent with the role of activation dependent phosphorylation in the $\mathrm{N}$-terminal activation segment of both PTKs and STKs, but suggests that the orientation of the C-terminal activation segment is more informative in the conformational classification of STKs. 


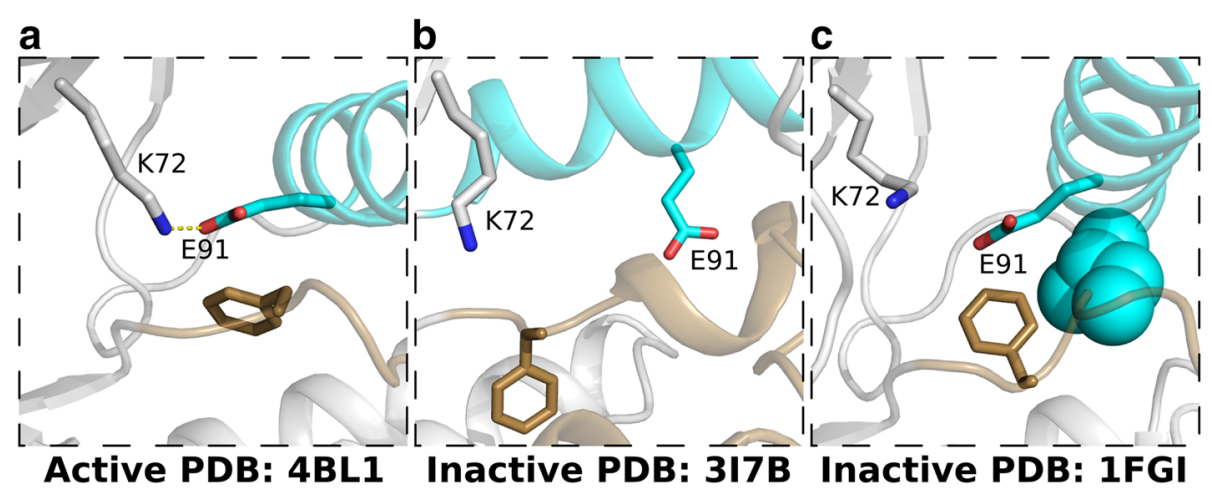

Fig. 7 Activation segment conformations. a Active conformation. b Inactive conformation with 1 1 1 t turn activation segment helix sterically blocking aC-helix movement. c Inactive conformation with DFG-Phe up sterically blocking formation of K-E salt bridge. The aC-helix is colored teal and the activation segment is colored brown

We can observe the differences in activation segment conformations in PTK and STK crystal structures (Fig. 9c). In the PTK EGFR, for example, we observe large conformational changes in the $\mathrm{N}$-terminal activation segment, with the $1 \frac{1}{1 / 2}$ turn helix blocking the $\alpha \mathrm{C}$-helix in the inactive conformation. However, only slight changes can be seen in the C-terminal activation segment of EGFR between the active and inactive conformations. In contrast, in STK structures such as ERK2, the N-terminal activation segment is less drastically altered than in EGFR, with only a slight rotation in the DFG-phenylalanine. The ERK2 inactive $\mathrm{C}$-terminal activation segment is markedly changed between the active and inactive conformations, however, with an uncoiled helix that partially blocks active site access. The necessity of incorporating $\mathrm{C}$-terminal activation segment features in STK classification may be due to the fact that STKs conserve phosphorylatable residues in the C-terminus of the activation segment [41-52]. In contrast, PTKs naturally conserve hydrophobic residues at the activation segment $\mathrm{C}$-terminus, providing a plausible explanation for the observed difference in PTK and STK features.

\section{CDK family feature selection identifies cyclin binding residues}

The cyclin-dependent kinase (CDK) family is a set of STKs involved in cell-cycle progression [53], replication stress response [54, 55] and transcription [56, 57]. CDK activity is, as its name suggests, dependent on the formation of a CDK-cyclin complex. Upon binding, cyclin

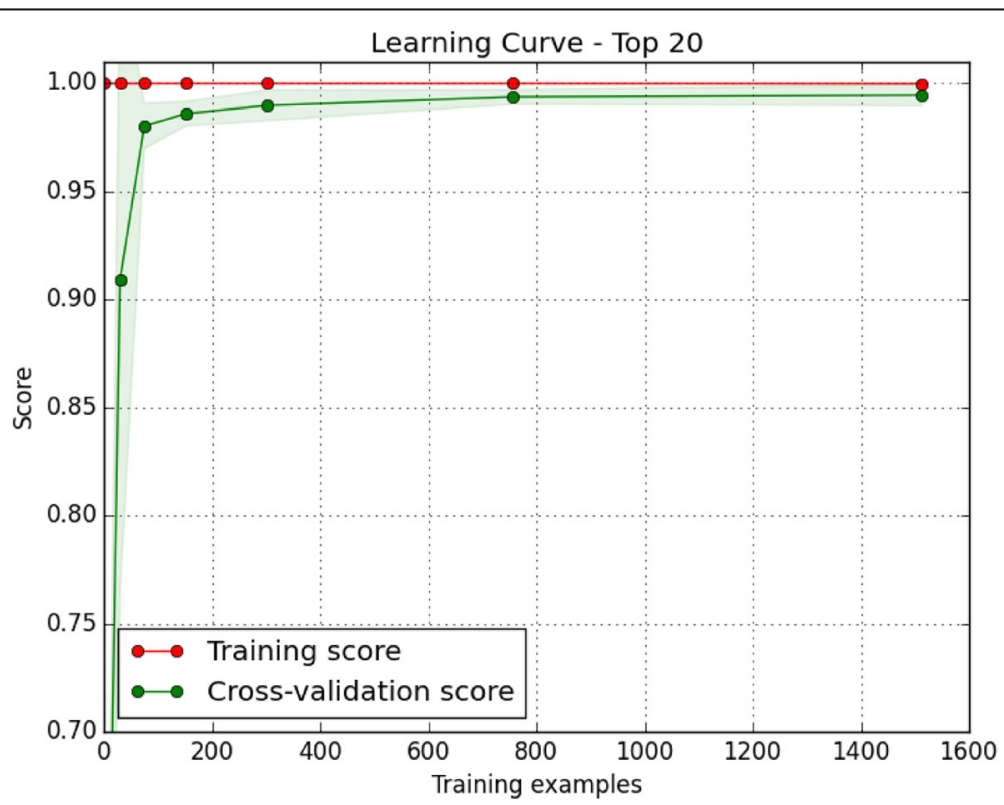

Fig. 8 Learning curve on ePK classifier. Learning curve on the ePK classifier for the top 20 selected features. With more than 200 training samples, we achieve high classification accuracy 
Table 3 Top 10 PTK selected features

\begin{tabular}{lllc}
\hline Feature & Activation segment location & PKA positions & Average rank \\
\hline Pd_140-143 & N-terminal & $187-190$ & 3.10 \\
Pd_137-140 & N-terminal & $184-187$ & 3.40 \\
Ф_141 & N-terminal & 188 & 8.75 \\
Pd_141-144 & N-terminal & $188-191$ & 11.08 \\
$\Psi$ _139 & N-terminal & 186 & 11.60 \\
Ф_143 & N-terminal & 190 & 13.42 \\
D_142 & N-terminal & 189 & 14.90 \\
Pd_139-142 & N-terminal & $186-189$ & 14.98 \\
Pd_138-141 & N-terminal & $185-188$ & 25.70 \\
Pd_135-138 & N-terminal & $182-185$ & 28.70 \\
\hline
\end{tabular}

induces conformational changes in the kinase domain that allow for autophosphorylation of the activation segment to produce a fully active kinase [39]. The CDK family also has the largest number of crystal structures deposited in the PDB, providing 514 chains for our feature selection process. As we previously described, STK (and thus, CDK) classification is based on both $\mathrm{N}$ - and $\mathrm{C}$ - terminal activation segments. However, CDKs are allosterically regulated through cyclin binding. To test whether our feature selection procedure would be able to identify the CDK-cyclin interactions, we removed the activation segment features from our CDK training samples and repeated feature selection on this subset. The most important features are found in the $\beta 3-\alpha C$ loop and the C-terminal segment of the $\alpha C$ helix, both of which are involved in the CDK-cyclin interface. Less significant features in the CDK-cyclin interface were identified in the $\beta 4-\beta 5$ loop. Two additional features were found that are distal to the cyclin binding site, one in the catalytic loop and the other in the $\alpha \mathrm{F}$ helix (Fig. 10).

\section{Inactive structures cluster by evolutionary history}

To identify patterns in the conformation of inactive structures we took an unsupervised approach, using principal component analysis (PCA) to identify the direction of greatest variance in our dataset. As PCA is an inherently linear process, we transformed the cyclical angle measures of the inactive structures to the Cartesian coordinates on the unit circle $(\theta \rightarrow(\sin \theta, \cos \theta)$. After limiting the dataset to the top weighted features in the first three principal components, we again performed PCA to ensure the clustering patterns were similar.

When we applied the above process to all ePK inactive structures, we discovered that the most heavily weighted features were not measuring backbone conformations with $\phi, \psi$, or pseudo-dihedral angles, but $\chi^{1}$ angles which measure side chain orientation (Table 6). The residues identified are spread throughout the kinase domain, occurring in both the $\mathrm{N}$ - and $\mathrm{C}$ - lobes, with several residues identified in the catalytic loop and activation segment. By plotting the structures in the principal component plane (PC1, PC2), we readily identified three clusters. Two of the clusters are kinase group

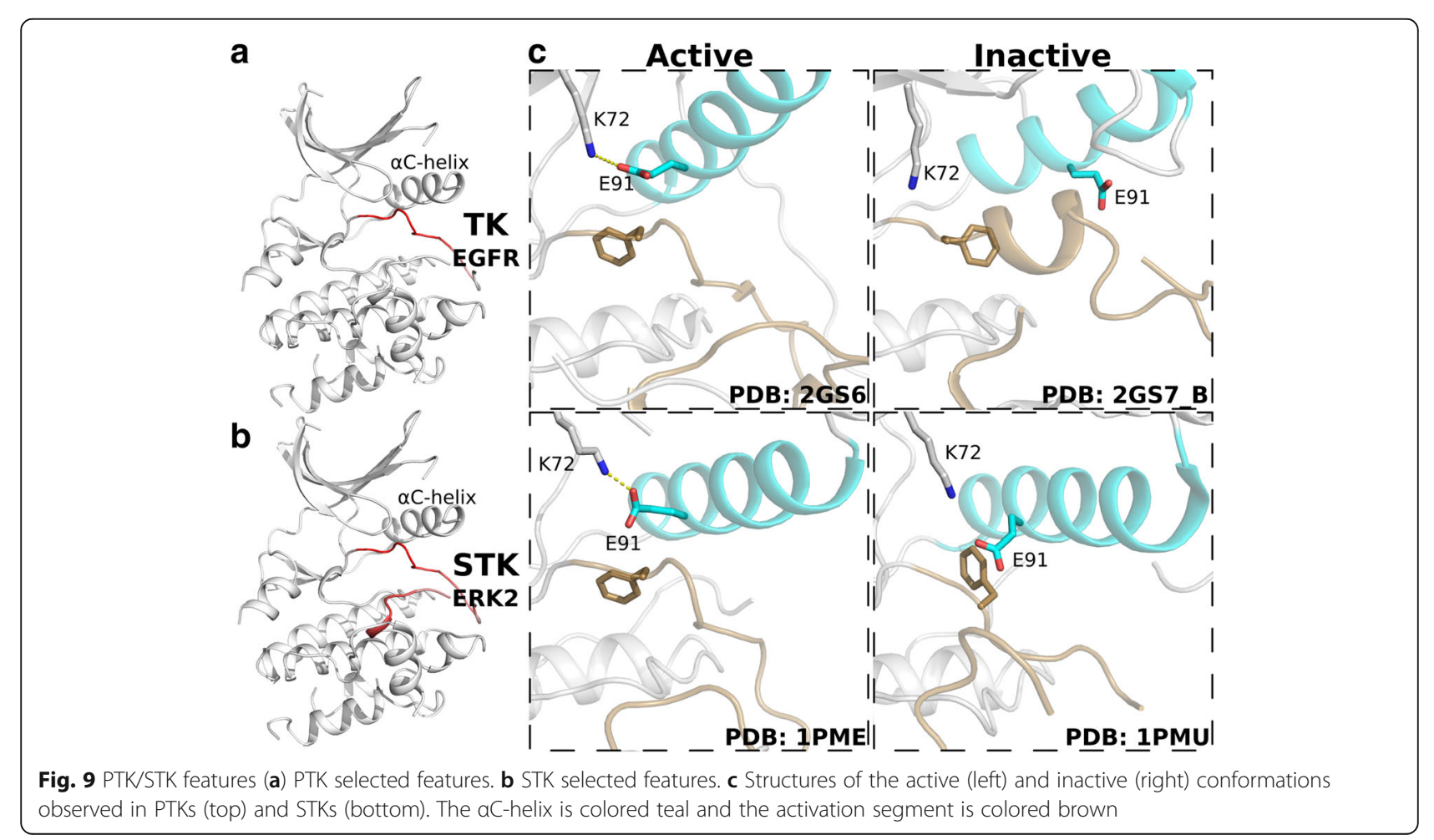


Table 4 Top 20 STK selected features

\begin{tabular}{|c|c|c|c|}
\hline Feature & Activation segment location & PKA positions & Average rank \\
\hline Pd_137-140 & N-terminal & $184-187$ & 3.20 \\
\hline$\Psi_{-} 141$ & $\mathrm{~N}$-terminal & 188 & 6.97 \\
\hline$\psi_{-} 142$ & $\mathrm{~N}$-terminal & 189 & 8.85 \\
\hline Pd_140-143 & N-terminal & $187-190$ & 11.25 \\
\hline Pd_155-158 & C-terminal & $200-203$ & 13.78 \\
\hline Pd_141-144 & $\mathrm{N}$-terminal & 188-191 & 15.07 \\
\hline U_138 & $\mathrm{N}$-terminal & 185 & 15.23 \\
\hline Ф_139 & N-terminal & 186 & 17.13 \\
\hline$\psi_{-} 144$ & $\mathrm{~N}$-terminal & 191 & 20.45 \\
\hline$\Psi_{-} 143$ & $\mathrm{~N}$-terminal & 190 & 21.98 \\
\hline Pd_139-142 & $\mathrm{N}$-terminal & $186-189$ & 29.35 \\
\hline Ф_155 & C-terminal & 200 & 30.13 \\
\hline Pd_138-141 & N-terminal & $185-188$ & 32.80 \\
\hline Pd_136-139 & N-terminal & 183-186 & 33.05 \\
\hline Ф_142 & N-terminal & 189 & 37.57 \\
\hline Pd_135-138 & N-terminal & $182-185$ & 38.42 \\
\hline U_139 & N-terminal & 186 & 43.98 \\
\hline$\Psi \_154$ & C-terminal & 199 & 46.17 \\
\hline Pd_156-159 & C-terminal & $201-204$ & 48.70 \\
\hline U_157 & C-terminal & 202 & 49.08 \\
\hline
\end{tabular}

Table 5 Top 20 non-CMGC STK selected features

\begin{tabular}{|c|c|c|c|}
\hline Feature & Activation segment location & PKA positions & Average rank \\
\hline Pd_137-140 & N-terminal & $184-187$ & 5.30 \\
\hline Pd_155-158 & C-terminal & $200-203$ & 15.23 \\
\hline Pd_136-139 & N-terminal & $183-186$ & 19.77 \\
\hline Ф_155 & C-terminal & 200 & 27.30 \\
\hline Pd_141-144 & N-terminal & 188-191 & 27.98 \\
\hline Pd_139-142 & N-terminal & 186-189 & 28.90 \\
\hline Ф_139 & N-terminal & 186 & 34.55 \\
\hline U_139 & $\mathrm{N}$-terminal & 186 & 34.83 \\
\hline$\psi_{-} 141$ & N-terminal & 188 & 38.37 \\
\hline Pd_140-143 & N-terminal & $187-190$ & 40.02 \\
\hline$\Psi_{-} 144$ & $\mathrm{~N}$-terminal & 191 & 40.65 \\
\hline Pd_135-138 & N-terminal & $182-185$ & 42.88 \\
\hline Pd_138-141 & $\mathrm{N}$-terminal & $185-188$ & 44.73 \\
\hline$\psi_{-} 154$ & C-terminal & 199 & 47.73 \\
\hline Ф_137 & $\mathrm{N}$-terminal & 184 & 48.65 \\
\hline Ф_155 & C-terminal & 200 & 49.75 \\
\hline Ф_154 & C-terminal & 199 & 53.75 \\
\hline$\psi_{-} 143$ & N-terminal & 190 & 59.55 \\
\hline U_138 & N-terminal & 185 & 61.13 \\
\hline U_156 & C-terminal & 201 & 65.42 \\
\hline
\end{tabular}

specific, separating PTKs and CMGCs from the remainder of kinases in the third cluster (Fig. 11a). This is consistent with group biases of several of the top weighted residues, like the Lys in the LKPEN STK specific motif, which is naturally conserved in PTKs as LAARN. Similarly, the STK specific phosphorylation site at $201^{\text {PKA }}$, which is conserved in PTKs as a hydrophobic residue, is heavily weighted in the principal components.

A similar pattern emerged when we considered only the PTK inactive structures. Again, we identify side chain conformations ( $X 1$ angles) as providing the greatest variance in the dataset. Here, the residues identified lie mostly in the C-lobe, occupying positions in the substrate binding region, $\mathrm{P}+1$ loop, and the activation segment (Table 6). Plotting the structures in the first three principal components reveals an analogous pattern, with clusters consisting of specific PTK families. The most distinct clusters separate the Abelson tyrosine-protein kinase (Abl) family, the Insulin receptor (InsR) family, the tyrosine-protein kinase Tec (Tec) family, and the hepatocyte growth factor receptor (Met) family (Fig. 11b). In contrast, the top weighted features in the CMGC group consist entirely of pseudo-dihedral angles (Table 6). The CMGC inactive structures are largely dominated by the CDK family, likely explaining the variance present in backbone measurements. Again, the features are mostly in the $\mathrm{C}$-lobe, occurring in the $\alpha \mathrm{D}$-helix, at the terminal ends of the $\alpha$ E-helix, and the $\alpha \mathrm{G}$ - and $\alpha \mathrm{H}$ - helices. The only $\mathrm{N}$ lobe feature identified is in the C-terminal portion of the $\alpha C$ - $\beta 4$ loop (Fig. 11c). To avoid issues with the side chain placement of low resolution structures, we repeated the above inactive clustering with only high resolution structures $(<2.2 \AA)$. Again, we find that the greatest source of variance in our inactive datasets correlates with the evolutionary relationships between kinases, with clusters similar to those in Fig. 11. Thus the conformational features appear to implicitly capture the evolutionary relationships between kinases, even though evolutionary features (sequence similarity, for example) are not explicitly considered in our analysis.

\section{Classifier available for public use}

To encourage consistency in kinase structure annotations, we have made our ePK random forest classifier publicly available through a stand-alone program located at https://github.com/esbg/kinconform [DOI:10.5281/ zenodo.249090], allowing users to annotate newly solved kinase PDB structures and/or simulated structures as active/inactive. The sequence for each chain is extracted and aligned to our highly curated profile alignments. The features necessary for classification are then measured and a prediction is generated. We have also included both our curated annotations for the training and validation sets, as well as predicted annotations on 


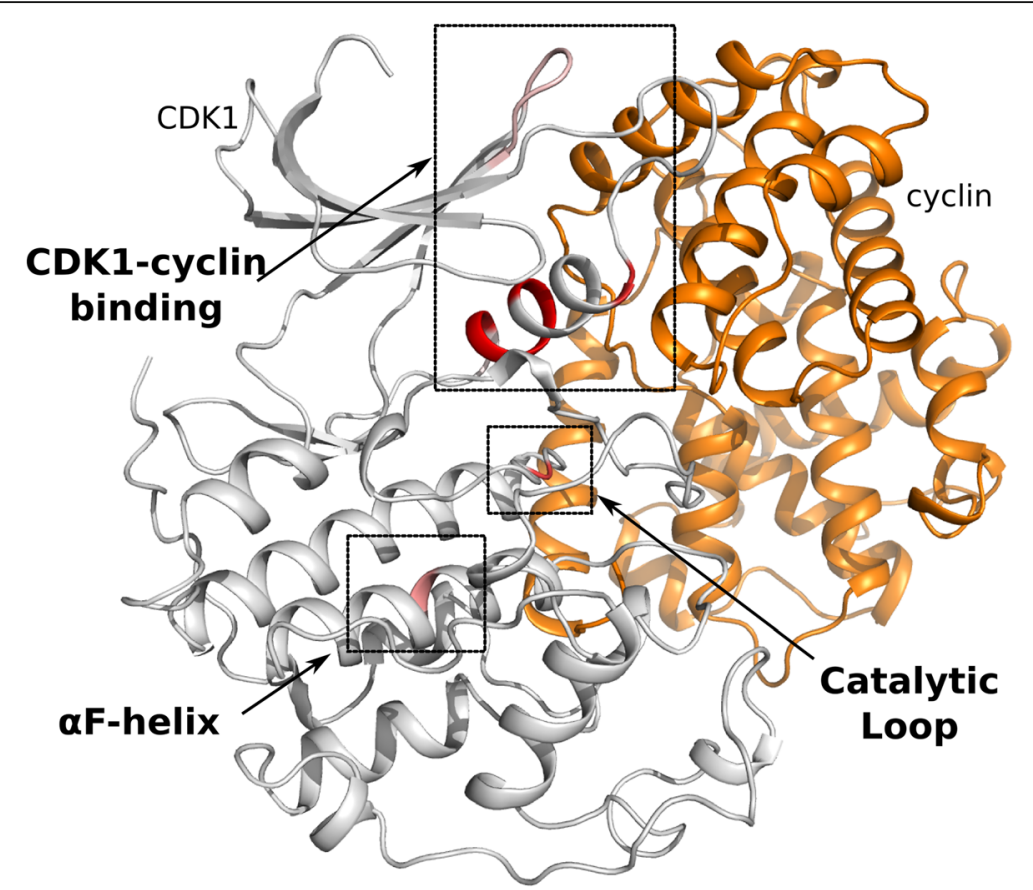

Fig. 10 CDK1 with selected features. We identify features in three regions: the cyclin binding interface, the catalytic loop, and the aF-helix. CDK1 is colored white, with identified features in red. Cyclin is colored orange

all kinase PDBs in the structure class of ProKinO, the Protein Kinase Ontology [36, 37]. Using the hasAnnotatedConformation and hasPredictedConformation data properties, users can easily identify which structures were used for training/validation, which structures were misclassified, as well as the predicted conformation for remaining structures. The dataset of measurements, predicted conformations and annotated conformations are included [see Additional files 1 and 2].

\section{Conclusion}

We have provided the first comprehensive machine learning based classification of protein kinase active/inactive conformations, taking into account more structures and measurements than any previous classification effort. The features identified in our analysis reflect previous knowledge about the conformation of the N-terminal activation segment, as well as provide new insights into the importance of the C-terminal activation segment in classifying STK structures. Given a sufficient number of evolutionarily related structures, we can also identify protein interfaces and regulatory regions, as shown with the cyclin-CDK1 complex. We show statistically significant correlations between activation segment orientation and $\alpha \mathrm{C}$ helix orientation, and suggest a classification scheme based on activation segment orientation alone. Further, our unbiased exploration of inactive structures has revealed that the greatest

Table 6 Top 10 features in inactive structures among all ePKs, PTKs, and CMGCs

\begin{tabular}{|c|c|c|c|c|c|}
\hline ePK features & PKA positions & TK features & PKA positions & CMGC features & PKA positions \\
\hline X1_121 & 168 & X1_166 & 211 & Pd_217-220 & $268-271$ \\
\hline X1_64 & 110 & X1_94 & 141 & Pd_56-59 & $102-105$ \\
\hline X1_79 & 125 & X1_197 & 242 & Pd_157-160 & $202-205$ \\
\hline X1_142 & 189 & X1_26 & 68 & Pd_202-205 & $247-250$ \\
\hline X1_100 & 147 & X1_150 & 195 & Pd_219-222 & $270-273$ \\
\hline X1_218 & 269 & X1_235 & 291 & Pd_229-232 & $280-283$ \\
\hline X1_29 & 71 & X1_187 & 232 & Pd_109-112 & 156-159 \\
\hline X1_132 & 179 & X1_160 & 205 & Pd_95-98 & $142-145$ \\
\hline X1_156 & 201 & X1_147 & N/A & Pd_199-202 & $244-247$ \\
\hline X1_131 & 178 & X1_52 & 98 & Pd_85-88 & $131-134$ \\
\hline
\end{tabular}



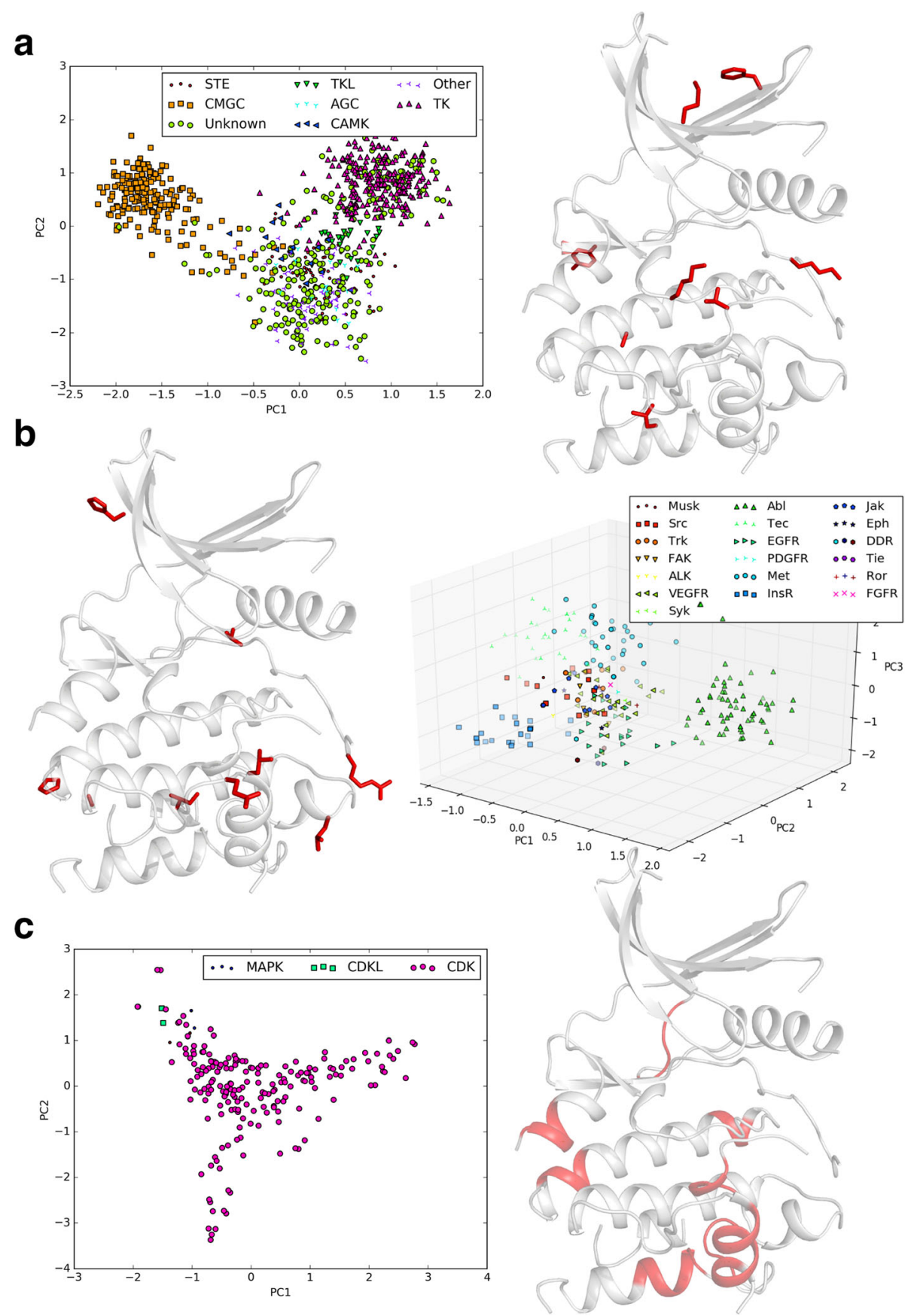

Fig. 11 Inactive clusters and features. a All ePKs. b PTK group. c CMGC group

variation between inactive conformations lies in kinase group and family specific side chain orientations. This is interesting given that the evolutionary relationships between kinases are not used as features in the classification.

While we strive to ensure the accuracy of our alignment profiles, the above analysis may be affected by alterations therein. Our methods are also highly dependent on the number of structures available, which is constantly increasing, and the initial set of annotations in the training set. In the future, semi-supervised methods may be beneficial in extending our initial set of curated annotations.

The techniques used above are not kinase specific, and can be applied to any protein family with a conserved fold, a sufficient number of deposited crystal structures, and a curated multiple sequence alignment. Further, while we explored the difference between active and inactive structures, the annotations provided could range 
over a variety of topics including, whether a ligand, substrate or regulatory molecule is bound (or unbound), the presence (or absence) of a post-translational modification, or any binary attribute of interest. Finally, the residues identified in our analysis can be used in the design of selective protein kinase inhibitors.

\section{Methods}

\section{Dataset construction}

We identified kinase structures in RCSB [58] through sequence alignment to a set of previously generated manually curated kinase profiles [59], yielding 3,488 PDBs with 5,131 chains. To establish our active/inactive annotations, we first classified each of the structures using previously published classification methods. They agreed on the conformational state for 3,098 of the 5,131 chains (60.4\%), which we labeled accordingly. Disagreements were settled through consensus manual curation by two independent biochemists, which resulted in sets of labeled and unlabeled chains, with 3,365 and 1,766 members, respectively. We further separated our labeled chains into two sets: a randomly selected training set of 1,000 chains (500 active, 500 inactive) used for feature selection, and a validation set containing the remaining 2,365 chains. This process is quite robust and was repeated 10 times with essentially identical features and classification accuracy. Given the small number of training samples needed to construct an accurate classifier (Fig. 8), one could also perform the initial annotation by selecting and manually curating structures randomly until a balanced dataset of sufficient size is generated.

\section{Feature construction}

For each chain, we created a unique vector which represents the conformation by measuring, the $\phi, \psi$ and $X^{1}$ angles at each aligned residue in our profile, creating $241 * 3=723$ features. In addition, we measured the pseudo-dihedral angle through the alpha carbon of adjacent quads of residues [22], incorporating an additional 238 features and bringing our total feature count to 961 . We do not consider pairwise distances between residues, as they would incorporate an additional 28,920 features, and greatly increase the likelihood of over fitting. Measurements were made using the MDAnalysis toolkit [60].

\section{Feature selection}

Feature selection was performed on our training set, consisting of 1,000 PDB chains with an average resolution of $2.2 \AA$. We used a variety of feature selection algorithms (OneR [61], chi-squared, ReliefF [62], Gain-Ratio [63], correlation-based feature selection [64]) with 10-fold cross validation to identify which of the 961 incorporated features are most informative in separating active and inactive structures. The four single-attribute evaluators provide a rank for each feature importance, which we averaged over all evaluators. As our features are numbered according to our profile alignment, we also mapped the identified features onto a template structure to identify their location within the kinase domain. Feature selection was performed with Weka v3.6.11 [65].

\section{Classifier construction and parameter optimization}

We used multiple classification algorithms to classify active from inactive structures, including naïve Bayes, neural network, random forest and support vector classifiers. Naïve Bayes classifiers are probabilistic classifiers which assume independence between the features and apply Bayes' theorem. Neural networks, inspired by biological neurons, consist of a collection of nodes (neurons) and edges (axons) that are trained on the input data. Support vector classifiers are nonprobabilistic and identify the hyper-plane that best partitions the high dimensional space in which the dataset resides. The most accurate classifier was generated using a random forest, which is an ensemble method utilizing a parameterized number of decision trees and outputting their mode as the classification. Each tree in the forest also uses a parameterized maximum number of features in making its decision. We performed a grid search of the parameter space to identify the optimal parameters for use with our selected features. Parameter searches, classifier construction, and PCA were performed in Python 2.7 [66], using the Scikit-learn machine learning toolkit [67]. Plots were made with Matplotlib 1.5.1 [68].

\section{Additional files}

Additional file 1: Predictions and curated annotations of kinase PDB files. This is three column CSV file which states the PDB ID and chain (column 1), the predicted annotation (column 2), and the curated annotation (column 3). (CSV $114 \mathrm{~kb}$ )

Additional file 2: Machine learning dataset. This file contains the measured $\Phi, \Psi, X_{1}$, and pseudo-dihedral angles used in the analysis. (ZIP $15492 \mathrm{~kb})$

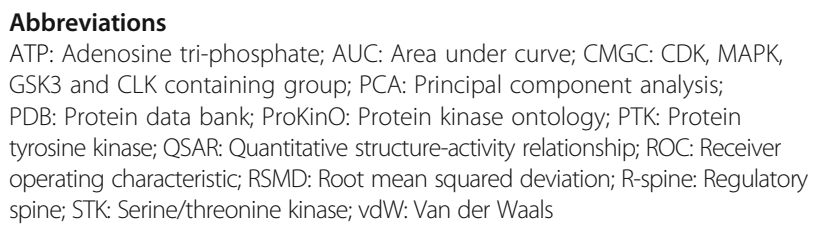

\section{Acknowledgements}

We thank Krishnadev Oruganty (GVKBIO) and Henrik Möbitz (Novartis) for assistance in curating our training set. 


\section{Availability of data and materials}

Our ePK classifier is publicly available through a stand-alone program located at https://github.com/esbg/kinconform [DOl:10.5281/zenodo.249090]. The dataset of measurements, predicted conformations and annotated conformations are included [see Additional files 1 and 2].

\section{Authors' contributions}

DIM, KR and NK designed the study. DIM collected data and performed analysis. DIM, KR and NK wrote, revised and approved the manuscript.

\section{Competing interests}

The authors declare that they have no competing interests.

\section{Consent for publication}

Not applicable.

\section{Ethics approval and consent to participate}

Not applicable.

\section{Author details}

${ }^{1}$ Institute of Bioinformatics, University of Georgia, Athens, GA 30602, USA. ${ }^{2}$ Department of Computer Science, University of Georgia, Athens, GA 30602, USA. ${ }^{3}$ Department of Biochemistry \& Molecular Biology, University of Georgia, Athens, GA 30602, USA.

\section{Received: 3 November 2016 Accepted: 28 January 2017} Published online: 02 February 2017

\section{References}

1. Hanks SK, Hunter T. Protein kinases 6. The eukaryotic protein kinase superfamily: kinase (catalytic) domain structure and classification. FASEB J. 1995;9(8):576-96.

2. Hanks SK, Quinn AM, Hunter T. The protein kinase family: conserved features and deduced phylogeny of the catalytic domains. Science (New York, NY). 1988;241:42-52.

3. Manning G, Whyte DB, Martinez R, Hunter T, Sudarsanam S. The protein kinase complement of the human genome. Science (New York, NY). 2002;298:1912-34.

4. Brown NR, Noble ME, Endicott JA, Johnson LN. The structural basis for specificity of substrate and recruitment peptides for cyclin-dependent kinases. Nat Cell Biol. 1999;1(7):438-43.

5. Endicott JA, Noble ME, Johnson LN. The structural basis for control of eukaryotic protein kinases. Annu Rev Biochem. 2012;81:587-613.

6. Johnson LN, Noble ME, Owen DJ. Active and inactive protein kinases: structural basis for regulation. Cell. 1996;85(2):149-58.

7. Nagar B, Bornmann WG, Pellicena P, Schindler T, Veach DR, Miller WT, Clarkson B, Kuriyan J. Crystal structures of the kinase domain of c-Abl in complex with the small molecule inhibitors PD173955 and imatinib (STI-571). Cancer Res. 2002;62(15):4236-43.

8. Schindler T, Bornmann W, Pellicena P, Miller WT, Clarkson B, Kuriyan J. Structural mechanism for STI-571 inhibition of abelson tyrosine kinase. Science. 2000;289(5486):1938-42.

9. Liu Y, Gray NS. Rational design of inhibitors that bind to inactive kinase conformations. Nat Chem Biol. 2006;2(7):358-64.

10. Wan PT, Garnett MJ, Roe SM, Lee S, Niculescu-Duvaz D, Good VM, Jones CM, Marshall CJ, Springer CJ, Barford D, et al. Mechanism of activation of the RAF-ERK signaling pathway by oncogenic mutations of B-RAF. Cell. 2004;116(6):855-67.

11. Pargellis C, Tong L, Churchill L, Cirillo PF, Gilmore T, Graham AG, Grob PM, Hickey ER, Moss N, Pav S, et al. Inhibition of p38 MAP kinase by utilizing a novel allosteric binding site. Nat Struct Biol. 2002;9(4):268-72.

12. Zheng J, Knighton DR, Ten Eyck LF, Karlsson R, Xuong NH, Taylor SS, Sowadski JM. Crystal structure of the catalytic subunit of CAMP-dependent protein kinase complexed with magnesium-ATP and peptide inhibitor Biochemistry. 1993;32(9):2154-61.

13. Madhusudan, Trafny EA, Xuong NH, Adams JA, Ten Eyck LF, Taylor SS, Sowadski JM. cAMP-dependent protein kinase: crystallographic insights into substrate recognition and phosphotransfer. Protein Sci. 1994;3(2):176-87.

14. Lowinger TB, Riedl B, Dumas J, Smith RA. Design and discovery of small molecules targeting raf-1 kinase. Curr Pharm Des. 2002;8(25):2269-78.
15. Cowan-Jacob SW, Fendrich G, Floersheimer A, Furet $P$, Liebetanz J, Rummel G, Rheinberger P, Centeleghe M, Fabbro D, Manley PW. Structural biology contributions to the discovery of drugs to treat chronic myelogenous leukaemia. Acta Crystallogr D Biol Crystallogr. 2007;63(Pt 1):80-93.

16. Weisberg E, Manley PW, Breitenstein W, Brüggen J, Cowan-Jacob SW, Ray A, Huntly B, Fabbro D, Fendrich G, Hall-Meyers E, et al. Characterization of AMN107, a selective inhibitor of native and mutant Bcr-Abl. Cancer Cell. 2005;7(2):129-41.

17. Naumann T, Matter H. Structural classification of protein kinases using 3D molecular interaction field analysis of their ligand binding sites: target family landscapes. J Med Chem. 2002;45(12):2366-78.

18. Kufareva I, Abagyan R. Type-II kinase inhibitor docking, screening, and profiling using modified structures of active kinase states. J Med Chem. 2008;51(24):7921-32.

19. Jacobs MD, Caron PR, Hare BJ. Classifying protein kinase structures guides use of ligand-selectivity profiles to predict inactive conformations: structure of Ick/imatinib complex. Proteins. 2008;70(4):1451-60.

20. Brooijmans N, Chang YW, Mobilio D, Denny RA, Humblet C. An enriched structural kinase database to enable kinome-wide structure-based analyses and drug discovery. Protein Sci. 2010;19(4):763-74.

21. van Linden OP, Kooistra AJ, Leurs R, de Esch IJ, de Graaf C. KLIFS: a knowledge-based structural database to navigate kinase-ligand interaction space. J Med Chem. 2014;57(2):249-77.

22. Möbitz $H$. The $A B C$ of protein kinase conformations. Biochim Biophys Acta. 2015:1854(10 Pt B):1555-66.

23. Kornev AP, Haste NM, Taylor SS, Eyck LF. Surface comparison of active and inactive protein kinases identifies a conserved activation mechanism. Proc Natl Acad Sci U S A. 2006;103(47):17783-8.

24. Meharena HS, Chang P, Keshwani MM, Oruganty K, Nene AK, Kannan N, Taylor SS, Kornev AP. Deciphering the Structural Basis of Eukaryotic Protein Kinase Regulation. PLoS Biol. 2013;11.

25. Battistutta R, Lolli G. Structural and functional determinants of protein kinase CK2a: facts and open questions. Mol Cell Biochem. 2011;356(1-2):67-73.

26. Bock JR, Gough DA. Predicting protein-protein interactions from primary structure. Bioinformatics. 2001;17(5):455-60.

27. Rost B, Sander C. Combining evolutionary information and neural networks to predict protein secondary structure. Proteins. 1994;19(1):55-72.

28. Muggleton S, King RD, Sternberg MJ. Protein secondary structure prediction using logic-based machine learning. Protein Eng. 1992;5(7):647-57.

29. Faraggi $E$, Kloczkowski A. A global machine learning based scoring function for protein structure prediction. Proteins. 2014;82(5):752-9.

30. Pires DE, de Melo-Minardi RC, dos Santos MA, da Silveira CH, Santoro MM, Meira W. Cutoff Scanning Matrix (CSM): structural classification and function prediction by protein inter-residue distance patterns. BMC Genomics. 2011;12:Suppl 4-S12.

31. Pires DE, de Melo-Minardi RC, da Silveira CH, Campos FF, Meira W. aCSM: noise-free graph-based signatures to large-scale receptor-based ligand prediction. Bioinformatics. 2013;29(7):855-61.

32. Fernandez-Lozano C, Cuiñas RF, Seoane JA, Fernández-Blanco E, Dorado J, Munteanu CR. Classification of signaling proteins based on molecular star graph descriptors using Machine Learning models. J Theor Biol. 2015;384:50-8.

33. Liu B, Zhang D, Xu R, Xu J, Wang X, Chen Q, Dong Q, Chou KC. Combining evolutionary information extracted from frequency profiles with sequencebased kernels for protein remote homology detection. Bioinformatics. 2014;30(4):472-9.

34. Weinkam $P$, Chen YC, Pons J, Sali A. Impact of mutations on the allosteric conformational equilibrium. J Mol Biol. 2013;425(3):647-61.

35. Jia L, Yarlagadda R, Reed CC. Structure Based Thermostability Prediction Models for Protein Single Point Mutations with Machine Learning Tools. PLoS One. 2015:10(9), e0138022.

36. Gosal GPS, Kannan N, Kochut KJ. ProKinO: A Framework for Protein Kinase Ontology. IEEE Int Conf Bioinformatics Biomed. 2011;2011:550-5.

37. Gosal G, Kochut KJ, Kannan N. ProKinO: an ontology for integrative analysis of protein kinases in cancer. PLoS One. 2011;6(12):e28782.

38. Jeffrey PD, Russo AA, Polyak K, Gibbs E, Hurwitz J, Massague J, Pavletich NP. Mechanism of CDK activation revealed by the structure of a cyclinA-CDK2 complex. Nature. 1995;376(6538):313-20.

39. Russo AA, Jeffrey PD, Pavletich NP. Structural basis of cyclin-dependent kinase activation by phosphorylation. Nat Struct Biol. 1996;3(8):696-700.

40. Yamaguchi $H$, Hendrickson WA. Structural basis for activation of human lymphocyte kinase Lck upon tyrosine phosphorylation. Nature. 1996;384(6608):484-9. 
41. Lee CH, Chung JH. The hCds1 (Chk2)-FHA domain is essential for a chain of phosphorylation events on hCds1 that is induced by ionizing radiation. J Biol Chem. 2001;276(32):30537-41.

42. Buscemi G, Perego P, Carenini N, Nakanishi M, Chessa L, Chen J, Khanna K, Delia D. Activation of ATM and Chk2 kinases in relation to the amount of DNA strand breaks. Oncogene. 2004;23(46):7691-700.

43. Cho J, Tsichlis PN. Phosphorylation at Thr-290 regulates Tpl2 binding to NFkappaB1/p105 and Tpl2 activation and degradation by lipopolysaccharide. Proc Natl Acad Sci U S A. 2005;102(7):2350-5.

44. Timm T, Balusamy K, Li X, Biernat J, Mandelkow E, Mandelkow EM. Glycogen synthase kinase (GSK) 3beta directly phosphorylates Serine 212 in the regulatory loop and inhibits microtubule affinity-regulating kinase (MARK) 2. J Biol Chem. 2008;283(27):18873-82.

45. Jiang T, Qiu Y. Interaction between Src and a C-terminal proline-rich motif of Akt is required for Akt activation. J Biol Chem. 2003;278(18):15789-93.

46. Lai S, Pelech S. Regulatory roles of conserved phosphorylation sites in the activation T-loop of the MAP kinase ERK1. Mol Biol Cell. 2016;27(6):1040-50.

47. Jung HS, Kim DW, Jo YS, Chung HK, Song JH, Park JS, Park KC, Park SH, Hwang $\mathrm{JH}$, Jo KW, et al. Regulation of protein kinase B tyrosine phosphorylation by thyroid-specific oncogenic RET/PTC kinases. Mol Endocrinol. 2005;19(11):2748-59.

48. Chen R, Kim O, Yang J, Sato K, Eisenmann KM, McCarthy J, Chen H, Qiu Y. Regulation of Akt/PKB activation by tyrosine phosphorylation. J Biol Chem. 2001;276(34):31858-62.

49. Pearce $L R$, Komander D, Alessi DR. The nuts and bolts of AGC protein kinases. Nat Rev Mol Cell Biol. 2010;11(1):9-22.

50. Huang WC, Chen JJ, Chen CC. C-Src-dependent tyrosine phosphorylation of IKKbeta is involved in tumor necrosis factor-alpha-induced intercellular adhesion molecule-1 expression. J Biol Chem. 2003;278(11):9944-52.

51. Konishi H, Tanaka M, Takemura Y, Matsuzaki H, Ono Y, Kikkawa U, Nishizuka Y. Activation of protein kinase $\mathrm{C}$ by tyrosine phosphorylation in response to H2O2. Proc Natl Acad Sci U S A. 1997;94(21):11233-7.

52. Tyler RK, Chu ML, Johnson H, McKenzie EA, Gaskell SJ, Eyers PA. Phosphoregulation of human Mps1 kinase. Biochem J. 2009;417(1):173-81.

53. Draetta $G$, Beach D. Activation of cdc2 protein kinase during mitosis in human cells: cell cycle-dependent phosphorylation and subunit rearrangement. Cell. 1988;54(1):17-26.

54. Yu DS, Zhao R, Hsu EL, Cayer J, Ye F, Guo Y, Shyr Y, Cortez D. Cyclin-dependent kinase 9 -cyclin $\mathrm{K}$ functions in the replication stress response. EMBO Rep. 2010;11(11):876-82.

55. Yu DS, Cortez D. A role for CDK9-cyclin K in maintaining genome integrity. Cell Cycle. 2011;10(1):28-32.

56. Fu TJ, Peng J, Lee G, Price DH, Flores O. Cyclin K functions as a CDK9 regulatory subunit and participates in RNA polymerase II transcription. J Biol Chem. 1999;274(49):34527-30.

57. Yang Z, Zhu Q, Luo K, Zhou Q. The 7SK small nuclear RNA inhibits the CDK9/ cyclin T1 kinase to control transcription. Nature. 2001;414(6861):317-22.

58. Berman HM, Westbrook J, Feng Z, Gilliland G, Bhat TN, Weissig H, Shindyalov IN, Bourne PE. The Protein Data Bank. Nucleic Acids Res. 2000;28(1):235-42.

59. Talevich E, Kannan N. Structural and evolutionary adaptation of rhoptry kinases and pseudokinases, a family of coccidian virulence factors. BMC Evol Biol. 2013;13:117-117

60. Michaud-Agrawal N, Denning E, Woolf TB, Beckstein O. MDAnalysis: a toolkit for the analysis of molecular dynamics simulations. J Comput Chem. 2011:32(10):2319-27.

61. Holte R. Very simple classification rules perform well on most commonly used datasets. Mach Learn. 1993;11:63-91.

62. Kira K, Rendell L. A practical approach to feature selection. In: International Conference on Machine Learning. 1992: 249-256

63. Witten I, Frank E, Hall M. Data Mining: Practical Machine Learning Tools and Techniques. 3rd ed. Amsterdam: Morgan Kaufmann; 2011.

64. Hall M. Correlation-based feature subset selection for machine learning. Hamilton: University of Waikato; 1999.

65. Hall M, Frank E, Holmes G, Pfahringer B, Reutemann P, Witten IH. The WEKA data mining software: an update. ACM SIGKDD Explor Newsletter. 2009;11(1):10-8.

66. Sanner MF. Python: a programming language for software integration and development. J Mol Graph Model. 1999;17(1):57-61.

67. Pedregosa F, Varoquaux G, Gramfort A, Michel V, Thirion B, Grisel O, Blondel M, Prettenhofer P, Weiss R, Dubourg V. Scikit-learn: Machine learning in Python. J Mach Learn Res. 2011;12:2825-30.

68. Barrett P, Hunter J, Miller JT, Hsu JC, Greenfield P. matplotlib-A Portable Python Plotting Package. In: Astronomical Data Analysis Software and Systems XIV, vol. 347. 2005. p. 91.

\section{Submit your next manuscript to BioMed Central and we will help you at every step:}

- We accept pre-submission inquiries

- Our selector tool helps you to find the most relevant journal

- We provide round the clock customer support

- Convenient online submission

- Thorough peer review

- Inclusion in PubMed and all major indexing services

- Maximum visibility for your research

Submit your manuscript at www.biomedcentral.com/submit 